\title{
A Uniqueness Theorem of the 3-Dimensional Acoustic Scattering Problem in a Shallow Ocean with a Fluid-like Seabed
}

\author{
Robert P. Gilbert \\ Department of Mathematical Sciences \\ University of Delaware, Newark, DE 19716, U.S.A. \\ Miao-jung Ou \\ Institute for Mathematics and its Applications, \\ University of Minnesota, Minneapolis, MN 55455, USA
}

\begin{abstract}
This paper shows that under the assumption of the out-going radiation conditions at infinity, the time-harmonic acoustic scattered field off a sound-soft solid in a shallow ocean with a fluid-like seabed is unique in $C^{2}\left(M_{1}\right) \cap C^{2}\left(M_{2}\right) \cap C\left(R_{h}^{3} \backslash \Omega\right)$. Here $M_{1}$ is the water part, $M_{2}$ the seabed, $R_{h}^{3}$ the waveguide and $\Omega$ the solid object. The associated modal problem is studied and a representation formula for the solution in terms of the Green's function is derived.
\end{abstract}

\section{Introduction}

The purpose of this paper is to generalize Xu's uniqueness theorem [3] for timeharmonic acoustic scattering in a uniform shallow ocean containing a scatterer to an ocean with a fluid-like seabed. The latter is modeled as a two-layered wave guide. The further extension to a multi-layered is immediate using the same analysis. In order to prove this theorem, we need some notation and a detailed description of the problem which will be treated in this section.

In Figure $1, M_{1}$ is the water column, $M_{2}$ the fluid-like sediment (basement), and $\Gamma$ their interface. A submersible occupying the region $\Omega$ is situated completely inside $M_{1}$. Let $\Omega_{\rho}$ be a vertical cylinder with radius $\rho$ big enough such that $\Omega$ is completely inside it ( see Figure 1). The portion of this cylinder in $M_{1}$ and $M_{2}$ is denoted by $\Omega_{\rho}^{M_{1}}$ and $\Omega_{\rho}^{M_{2}}$, respectively. $C_{\rho}^{M_{1}}$ and $C_{\rho}^{M_{2}}$ are the lateral surfaces of these two sub-cylinders. $\Gamma_{\rho}^{0}$ is the top of $\Omega_{\rho}^{M_{1}}, \Gamma_{\rho}^{d}$ the top of $\Omega_{\rho}^{M_{2}}$ and $\Gamma_{\rho}^{h}$ is the 


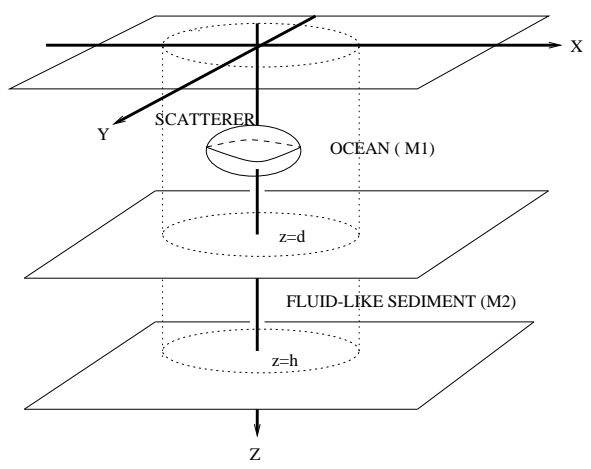

Figure 1: Schematic of the two-layered model.

bottom part of $\Omega_{\rho}^{M_{2}}$. The remaining notation is self explanatory. The following notation will be used throughout this chapter.

$$
\begin{aligned}
R_{h}^{3} & :=M_{1} \cup \Gamma \cup M_{2}, \\
D_{\varepsilon}(x, z) & :=\left\{(\xi, \zeta) \mid \sqrt{|x-\xi|^{2}+(z-\zeta)^{2}}<\varepsilon\right\} .
\end{aligned}
$$

The time-harmonic acoustic scattering is described in terms of the acoustic pressure $p$. In the water column and the basement, $p$ satisfies the differential equations

$$
\begin{aligned}
\triangle p(x, z)+k_{1}^{2} p(x, z) & =0,(x, z) \in M_{1} \backslash \bar{\Omega}, \\
\triangle p(x, z)+k_{2}^{2} p(x, z) & =0,(x, z) \in M_{2},
\end{aligned}
$$

respectively. Here $k_{j}$ is the wave number in $M_{j}, j=1,2$.

At the surface of the water column, we have a pressure release condition,

$$
p(x, 0)=0 .
$$

Across the interface of the water column and the basement, we have conservation of flux and pressure, namely

$$
\begin{aligned}
\frac{1}{\rho_{1}} \frac{\partial}{\partial z} p\left(x, d^{-}\right) & =\frac{1}{\rho_{2}} \frac{\partial}{\partial z} p\left(x, d^{+}\right), \\
p\left(x, d^{-}\right) & =p\left(x, d^{+}\right),
\end{aligned}
$$

where $\rho_{j}$ is the density in $M_{j}, j=1,2$ and the plus (minus) supscript represents the limit when approaching $\Gamma$ from the region $z>d(z<d)$. 
We assume a hard sub-sediment at $z=h$ and a boundary condition of Dirichlet type on the surface of the scatterer, i.e.

$$
\begin{aligned}
& \frac{\partial}{\partial z} p(x, h)=0, \\
& \left.p\right|_{\partial \Omega}=g(x, z) .
\end{aligned}
$$

It is well known that outside a cylinder of radius $\rho$, where $\rho$ is big enough such that $\Omega \subset \subset \Omega_{\rho}^{M_{1}}$, the pressure $p(x, z)$ has a normal mode representation

$$
p(x, z)=\sum_{n=0}^{\infty} \hat{p}_{n}(x, z)=\sum_{n=0}^{\infty} \psi_{n}(z) p_{n}(x), \quad \text { for }|x| \geq \rho .
$$

The $\psi_{n}(z)$ comes about by a separation of variables argument, which will be made clear in Section 3.

Since $R_{h}^{3}$ is an unbounded region, suitable radiation conditions should be posed at infinity.

$$
\lim _{r \rightarrow \infty} r^{1 / 2}\left(\frac{\partial p_{n}}{\partial r}-i a_{n} p_{n}\right)=0, n=0,1,2, \cdots, \infty
$$

where $r:=|x|$. Here $a_{n}$ is the $n^{\text {th }}$ eigenvalue of a system, which will be described in the next section. Physically, these conditions mean there is no incoming wave at infinity.

The paper is organized as follows. We started with the analysis of the modal problem in section 2. In section 3, the normal modes representation of the Green's function is given. Using the results in section 2 and section 3, a representation formula for the solution $p$ is derived in section 4 . The uniqueness theorem is then stated and proved in section 5 .

\section{Modal eigenvalue problem}

Recall that the Hankel transform $\tilde{f}(a)$ of a function $f(r)$ is defined as

$$
\tilde{f}(a)=2 \pi \int_{0}^{\infty} J_{0}(a r) f(r) r d r
$$

and the inverse Hankel transform is as

$$
f(r)=\frac{1}{2 \pi} \int_{0}^{\infty} J_{0}(a r) \tilde{f}(a) a d a .
$$


The modal eigenvalue problem is derived by applying the Hankel transformation with parameter $a$ to the system of (1)-(6) without the object $\Omega$. Denoting the Hankel transformation of the pressure function $p$ by $\tilde{\psi}$, the modal eigenvalue problem reads

$$
\begin{aligned}
\frac{d^{2}}{d z^{2}} \tilde{\Psi}(a, z)+\tau_{1}^{2} \tilde{\Psi}(a, z) & =0,0<z<d, \\
\frac{d^{2}}{d z^{2}} \tilde{\Psi}(a, z)+\tau_{2}^{2} \tilde{\Psi}(a, z) & =0, d<z<h, \\
\tilde{\Psi}(0) & =0, \\
\tilde{\psi}\left(a, d^{-}\right) & =\tilde{\psi}\left(a, d^{+}\right), \\
\frac{1}{\rho_{1}} \frac{d}{d z} \tilde{\psi}\left(a, d^{-}\right) & =\frac{1}{\rho_{2}} \frac{d}{d z} \tilde{\psi}\left(a, d^{+}\right), \\
\frac{d}{d z} \tilde{\psi}(a, h) & =0 .
\end{aligned}
$$

Here we have introduced the notation $\tau_{1}:=\sqrt{k_{1}^{2}-a^{2}}$ and $\tau_{2}:=\sqrt{k_{2}^{2}-a^{2}}$.

By a direct calculation, we get the characteristic equation of this system

$$
\tau_{1} \rho_{2} \cos \left(\tau_{1} d\right) \cos \left(\tau_{2}(h-d)\right)=\rho_{1} \tau_{2} \sin \left(\tau_{1} d\right) \sin \left(\tau_{2}(h-d)\right) .
$$

It can easily be checked using integration by parts that an appropriate inner product for this eigenfunction space is

$$
<\tilde{\psi}_{n}, \tilde{\Psi}_{m}>_{*}:=\int_{0}^{d} \tilde{\psi}_{n} \bar{\Psi}_{m} d z+\frac{\rho_{1}}{\rho_{2}} \int_{d}^{h} \tilde{\psi}_{n} \bar{\Psi}_{m} d z
$$

The induced norm is denoted by $\|\cdot\|_{*}$.

Lemma 2.1. There are countably many eigenvalues $a_{n}$ and there exists an $N \in \mathcal{N}$ such that $a_{0}^{2}>a_{1}^{2}>\ldots>a_{N}^{2}>0>a_{N+1}^{2}>\cdots$

Proof. The countability, simplicity and discreteness of the eigenvalues can easily be seen from the trigonometric characteristic equation (16). So it suffices to prove that the positive eigenvalues $\left\{a_{n}\right\}$ are bounded from above.

This can easily be proved by considering the characteristic equation (16). The imaginary part of the left-hand side of (16) is positive while that of the righthand side is negative for all $a>\max \left(k_{1}, k_{2}\right)$, so $a_{n}$ must be bounded above by $\max \left(k_{1}, k_{2}\right)$.

The following lemmas can be proved by using Sturm-Liouville type of argument to the modal eigenvalue problem [1] pp.63-66. 
Lemma 2.2. The square of the eigenvalues are real, namely

$$
a_{n}^{2} \in \boldsymbol{R}, \forall n
$$

In the remainder of this chapter, we shall choose $k_{1}, k_{2}$ such that $a_{n} \neq 0, \forall n=$ $0,1,2, \ldots$ and take $a_{n}>0$ if $a_{n}^{2}>0$ and $\operatorname{Im} a_{n}>0$ if $a_{n}^{2}<0$.

Lemma 2.3. Let $\tilde{\psi}_{n}$ and $\tilde{\psi}_{m}$ represent the eigenfunctions corresponding to the different eigenvalues $a_{n}$ and $a_{m}$, then

$$
<\tilde{\psi}_{n}, \tilde{\Psi}_{m}>_{*}=0 \text {. }
$$

Lemma 2.4. Let $\tilde{\Psi}_{n}$ and $\tilde{\Psi}_{m}$ represent the eigenfunctions corresponding to the different eigenvalues $a_{n}$ and $a_{m}$, then

$$
\int_{0}^{d} \tilde{\psi}_{n}(z) \tilde{\psi}_{m}(z) d z+\frac{\rho_{1}}{\rho_{2}} \int_{d}^{h} \tilde{\psi}_{n}(z) \tilde{\psi}_{m}(z) d z=0 .
$$

\section{Normal mode representation of the Green's function}

Let $\tilde{G}\left(a, z, z_{s}\right)$ denote the Hankel transform of the Green's function $G\left(x, z ; x_{s}, z_{s}\right)$ for the wave guide without the object. Assume that the transformation parameter is $a$, and $\left(x_{s}, z_{s}\right)$ denotes the source location. Note that in cylindrical coordinates the Greens function may be written as $G\left(x, z ; x_{s}, z_{s}\right)=G\left(\left|x-x_{s}\right|, z, z_{s}\right)=G\left(r, z, z_{s}\right)$, where $r=\left|x-x_{s}\right|$.

Furthermore, $\tilde{G}$ satisfies the transformed system

$$
\begin{aligned}
\frac{\partial^{2}}{\partial z^{2}} \tilde{G}\left(a, z, z_{s}\right)+\tau_{1}^{2} \tilde{G}\left(a, z, z_{s}\right) & =-\delta\left(z-z_{s}\right), 0<z<d, \\
\frac{\partial^{2}}{\partial z^{2}} \tilde{G}\left(a, z, z_{s}\right)+\tau_{2}^{2} \tilde{G}(a, z) & =0, d<z<h, \\
\tilde{G}(a, 0) & =0, \\
\tilde{G}\left(a, d^{-}\right) & =\tilde{G}\left(a, d^{+}\right), \\
\frac{1}{\rho_{1}} \frac{d}{d z} \tilde{G}\left(a, d^{-}\right) & =\frac{1}{\rho_{2}} \frac{d}{d z} \tilde{G}\left(a, d^{+}\right), \\
\frac{d}{d z} \tilde{G}(a, h) & =0,
\end{aligned}
$$

when $\left(x_{s}, z_{s}\right) \in M_{1}$. If $\left(x_{s}, z_{s}\right) \in M_{2}$, then we just change the right-hand side of (18) to be 0 and that of (19) to be $-\delta\left(z-z_{s}\right)$. We continue to use the notation $\tau_{1}=\sqrt{k_{1}^{2}-a^{2}}$ and $\tau_{2}=\sqrt{k_{2}^{2}-a^{2}}$. 
$\tilde{G}\left(a, z, z_{s}\right)$ may be constructed as follows: first, we find two linearly independent solutions of the homogeneous equations corresponding to equation (18) and (19) that satisfy both (21)and (22), and one solution satisfies (20) while the other satisfies (23). We shall denote the solution by $\tilde{\Psi}_{1}(a, z)$ and $\tilde{\Psi}_{2}(a, z)$, respectively. Then set

$$
\tilde{G}\left(a, z, z_{s}\right)=-\frac{\tilde{\Psi}_{1}\left(a, z_{<}\right) \tilde{\Psi}_{2}\left(a, z_{>}\right)}{W\left(\tilde{\Psi}_{1}, \tilde{\Psi}_{2}\right)\left(a, z_{s}\right)}
$$

where

$$
\begin{aligned}
& z_{<}:=\min \left(z, z_{s}\right) \\
& z_{>}:=\max \left(z, z_{s}\right),
\end{aligned}
$$

and

$$
W\left(\tilde{\Psi}_{1}, \tilde{\Psi}_{2}\right)=\operatorname{det}\left|\begin{array}{ll}
\tilde{\Psi}_{1}^{\prime} & \tilde{\Psi}_{2}^{\prime} \\
\tilde{\Psi}_{1} & \tilde{\Psi}_{2}
\end{array}\right|
$$

Now $\tilde{\Psi}_{1}(a, z)$ and $\tilde{\Psi}_{2}(a, z)$ can be obtained explicitly as:

$$
\tilde{\Psi}_{1}(a, z)=\left\{\begin{aligned}
\rho_{1} \tau_{2} \sin \left(\tau_{1} z\right) & , \quad 0<z<d \\
A_{1} \cos \left(\tau_{2} z\right)+B_{1} \sin \left(\tau_{2} z\right) & , \quad d<z<h
\end{aligned}\right.
$$

where

$$
\begin{gathered}
\left(\begin{array}{c}
A_{1} \\
B_{1}
\end{array}\right)=\left(\begin{array}{ll}
\rho_{1} \tau_{2} \cos \left(\tau_{2} d\right) \sin \left(\tau_{1} d\right)-\rho_{2} \tau_{1} \cos \left(\tau_{1} d\right) \sin \left(\tau_{2} d\right) \\
\rho_{1} \tau_{2} \sin \left(\tau_{2} d\right) \sin \left(\tau_{1} d\right)+\rho_{2} \tau_{1} \cos \left(\tau_{1} d\right) \cos \left(\tau_{2} d\right)
\end{array}\right), \\
\tilde{\Psi}_{2}(a, z)=\left\{\begin{array}{rr}
A_{2} \cos \left(\tau_{2} z\right)+B_{2} \sin \left(\tau_{1} z\right) & , 0<z<d, \\
\rho_{2} \tau_{1} \cos \left(\tau_{2}(h-z)\right) & , \quad d<z<h,
\end{array}\right.
\end{gathered}
$$

and where

$$
\left(\begin{array}{c}
A_{2} \\
B_{2}
\end{array}\right)=\left(\begin{array}{l}
\rho_{2} \tau_{1} \cos \left(\tau_{1} d\right) \cos \left(\tau_{2}(h-d)\right)-\rho_{1} \tau_{2} \sin \left(\tau_{1} d\right) \sin \left(\tau_{2}(h-d)\right) \\
\rho_{2} \tau_{1} \sin \left(\tau_{2} d\right) \cos \left(\tau_{1}(h-d)\right)+\rho_{1} \tau_{2} \cos \left(\tau_{1} d\right) \sin \left(\tau_{2}(h-d)\right)
\end{array}\right) .
$$

It is not surprising that $W\left(\tilde{\Psi}_{1}, \tilde{\Psi}_{2}\right)\left(a, z_{s}\right)$ is independent of $z_{s}$ and $W\left(\tilde{\Psi}_{1}, \tilde{\Psi}_{2}\right)=0$ is exactly the characteristic equation of section 2 . Note that the simple poles of $\tilde{G}\left(a, z, z_{s}\right)$ as a function of $a$ are the eigenvalues of the modal eigenvalue problem . Recall that $\left\{\psi_{n}(z)\right\}$ are the corresponding eigenfunctions. 
Applying the residue theorem to the inverse Hankel integral, we have the following representation for $G\left(r, z, z_{s}\right)$ [4]

$$
G\left(r, z, z_{s}\right)=-\frac{i}{4} \sum_{n=0}^{\infty} \psi_{n}(z) \psi_{n}\left(z_{s}\right) H_{0}^{(1)}\left(a_{n} r\right)
$$

where

$$
\begin{aligned}
\psi_{n}(z): & =\frac{\tilde{\Psi}\left(a_{n}, z\right)}{\left\|\tilde{\psi}\left(a_{n}, \cdot\right)\right\|_{*}} \\
\left\|\tilde{\psi}\left(a_{n}, \cdot\right)\right\|_{*}^{2}: & =\int_{0}^{d}\left|\tilde{\psi}_{1}\right|^{2}\left(a_{n}, z\right) d z+\int_{d}^{h} \frac{\rho_{1}}{\rho_{2}}\left|\tilde{\Psi}_{1}\right|^{2}\left(a_{n}, z\right) d z
\end{aligned}
$$

and $H_{0}^{(1)}$ is the zero order Hankel function of the first kind.

\section{A representation formula}

Let $\Omega_{\rho}$ be a right cylinder with the top at $z=0$, the bottom at $z=h$ and radius $\rho$ big enough so the scatterer is contained in it. $\Omega_{\rho}$ is divided into two sub-cylinders, $\Omega_{\rho}^{M_{1}}$ and $\Omega_{\rho}^{M_{2}}$, see Figure 1 .

For $(x, z) \in \Omega_{\rho}^{M_{1}}$, using integration by parts and applying the boundary conditions and transmission conditions, we have

$$
\begin{aligned}
0= & \frac{1}{\rho_{1}} \int_{C_{\rho}^{M_{1}}}\left[G(\xi, \zeta ; x, z) \frac{\partial p}{\partial v}(\xi, \zeta)-p \frac{\partial G}{\partial v}\right] d \sigma \\
& +\frac{1}{\rho_{2}} \int_{C_{\rho}^{M_{2}}} G \frac{\partial p}{\partial v}-p \frac{\partial G}{\partial v} d \sigma-\frac{1}{\rho_{1}} \int_{\partial \Omega} G \frac{\partial p}{\partial v}-p \frac{\partial G}{\partial v} d \sigma \\
& -\frac{1}{\rho_{1}} \int_{\partial D_{\varepsilon}(x, z)} G \frac{\partial p}{\partial v}-p \frac{\partial G}{\partial v} d \sigma \\
& +\left\{\int_{\Gamma_{\rho}^{d^{-}}} \frac{1}{\rho_{1}} G \frac{\partial p}{\partial z}-\frac{1}{\rho_{1}} u \frac{\partial G}{\partial z} d \sigma-\int_{\Gamma_{\rho}^{d^{+}}} \frac{1}{\rho_{2}} G \frac{\partial p}{\partial z}+\rho_{2} u \frac{\partial G}{\partial z} d \sigma\right\} .
\end{aligned}
$$


Similarly, for $(x, z) \in \Omega_{\rho}^{M_{2}}$, we get another identity

$$
\begin{aligned}
0= & \frac{1}{\rho_{1}} \int_{C_{\rho}^{M_{1}}} G \frac{\partial u}{\partial v}-p \frac{\partial G}{\partial v} d \sigma \\
& +\frac{1}{\rho_{2}} \int_{C_{\rho}^{M_{2}}} G \frac{\partial p}{\partial v}-p \frac{\partial G}{\partial v} d \sigma-\frac{1}{\rho_{1}} \int_{\partial \Omega} G \frac{\partial p}{\partial v}-p \frac{\partial G}{\partial v} d \sigma \\
& -\frac{1}{\rho_{2}} \int_{\partial D_{\varepsilon}(x, z)} G \frac{\partial p}{\partial v}-p \frac{\partial G}{\partial v} d \sigma \\
& +\left\{\int_{\Gamma_{\rho}^{d^{-}}} \frac{1}{\rho_{1}} G \frac{\partial p}{\partial z}-\frac{1}{\rho_{1}} p \frac{\partial G}{\partial z} d \sigma-\int_{\Gamma_{\rho}^{d^{+}}} \frac{1}{\rho_{2}} G \frac{\partial p}{\partial z}-\frac{1}{\rho_{2}} p \frac{\partial G}{\partial z} d \sigma\right\}
\end{aligned}
$$

Moreover, writing $\delta:=\sqrt{|\xi-x|^{2}+(\zeta-z)^{2}}$, we have [4]

$$
G(\xi, \zeta ; x, z)=-\frac{1}{4 \pi \delta}+O(1) \quad \text { as } \quad \delta \longrightarrow 0 .
$$

Letting $\varepsilon \longrightarrow 0$ in (27) and (28), by considering (29) and applying the mean value theorem, we obtain

$$
p(x, z)=\left\{\begin{aligned}
& \int_{\partial \Omega}\left[p(\xi, \zeta) \frac{\partial G}{\partial v}(x, z ; \xi, \zeta)-G(x, z ; \xi, \zeta) \frac{\partial p}{\partial v}(\xi, \zeta)\right] d \sigma \\
+ & \int_{C_{\rho}^{M_{1}}}\left[G(\xi, \zeta ; x, z) \frac{\partial p}{\partial v}(\xi, \zeta)-p(x, z) \frac{\partial G}{\partial v}(x, z ; \xi, \zeta)\right] d \sigma \\
+ & \frac{\rho_{1}}{\rho_{2}} \int_{C_{\rho}^{M_{2}}}\left[G(x, z ; \xi, \zeta) \frac{\partial p}{\partial v}(x, z)-p(x, z) \frac{\partial G}{\partial v}(x, z ; \xi, \zeta)\right] d \sigma \\
& (x, z) \in \Omega_{\rho}^{M_{1}} \backslash \bar{\Omega}, \\
& \frac{\rho_{2}}{\rho_{1}} \int_{\partial \Omega}\left[G(x, z ; \xi, \zeta) \frac{\partial p}{\partial v}(x, z)-p(x, z) \frac{\partial G}{\partial v}(x, z ; \xi, \zeta)\right] d \sigma \\
+ & \frac{\rho_{2}}{\rho_{1}} \int_{C_{\rho}^{M_{1}}}\left[G(\xi, \zeta ; x, z) \frac{\partial p}{\partial v}(\xi, \zeta)-p(x, z) \frac{\partial G}{\partial v}(x, z ; \xi, \zeta)\right] d \sigma \\
+ & \int_{C_{\rho}^{M_{2}}}\left[G(x, z ; \xi, \zeta) \frac{\partial p}{\partial v}(x, z)-p(x, z) \frac{\partial G}{\partial v}(x, z ; \xi, \zeta)\right] d \sigma \\
& (x, z) \in \Omega_{\rho}^{M_{2}} .
\end{aligned}\right.
$$

Lemma 4.1.

$$
\int_{C_{\rho}^{M \alpha}}|p|^{2} d \sigma=O(1) \text { as } \rho \longrightarrow \infty, \alpha=1,2
$$


Proof. First, we want to show

$$
\sum_{n=0}^{N} \int_{C_{\rho}^{M \alpha}}\left|\hat{p}_{n}\right|^{2}=O(1) \text { as } \rho \longrightarrow \infty, \alpha=1,2 .
$$

Note that for $0 \leq n \leq N$, we have $a_{n}>0$ and the out-going radiation conditions (9) imply

$$
\begin{aligned}
0 & =\lim _{\rho \rightarrow \infty} \int_{C_{\rho}^{M \alpha}}\left|\frac{\partial \hat{p}_{n}}{\partial v}-i a_{n} \hat{p}_{n}\right|^{2} d \sigma \\
& =\lim _{\rho \rightarrow \infty} \int_{C_{\rho}^{M \alpha}}\left[\left|\frac{\partial \hat{p}_{n}}{\partial v}\right|^{2}+a_{n}^{2}\left|\hat{p}_{n}\right|^{2}+2 \operatorname{Im}\left\{a_{n} \hat{p}_{n} \frac{\overline{\partial \hat{p}_{n}}}{\partial v}\right\}\right] d \sigma, \\
\alpha=1,2 . &
\end{aligned}
$$

We now consider the integral

$$
\begin{aligned}
& \int_{\Omega_{\rho}^{M_{1}} \backslash \Omega}\left\{\hat{p}_{n} \triangle \overline{\hat{p}_{n}}+\nabla \hat{p}_{n} \nabla \overline{\hat{p}_{n}}\right\} d V \\
= & \int_{C_{\rho}^{M_{1}}} \hat{p}_{n} \frac{\overline{\partial \hat{p}_{n}}}{\partial \nu} d \sigma+\int_{\Gamma_{\rho}^{d^{-}}} \hat{p}_{n} \frac{\overline{\partial \hat{p}_{n}}}{\partial \nu} d \sigma+\int_{\partial \Omega} \hat{p}_{n} \frac{\overline{\partial \hat{p}_{n}}}{\partial v} d \sigma .
\end{aligned}
$$

On the other hand, by using (1), we have

$$
\int_{\Omega_{\rho}^{M_{1}} \backslash \Omega}\left\{\hat{p}_{n} \triangle \overline{\hat{p}_{n}}+\nabla \hat{p}_{n} \nabla \overline{\hat{p}_{n}}\right\} d V=\int_{\Omega_{\rho}^{M_{1}} \backslash \Omega}\left\{k_{1}^{2}\left|\hat{p}_{n}\right|^{2}+\left|\nabla \hat{p}_{n}\right|^{2}\right\} d V \in \boldsymbol{R} .
$$

Comparing (34) and (35), we realize

$$
\operatorname{Im}\left\{\int_{C_{\rho}^{M_{1}}} \hat{p}_{n} \frac{\overline{\partial \hat{p}_{n}}}{\partial v} d \sigma\right\}=\operatorname{Im}\left\{-\int_{\Gamma_{\rho}^{d^{-}}} \hat{p}_{n} \frac{\overline{\partial \hat{p}_{n}}}{\partial v} d \sigma-\int_{\partial \Omega} \hat{p}_{n} \frac{\overline{\partial \hat{p}_{n}}}{\partial v} d \sigma\right\} .
$$

Using similar reasoning with respect to the cylinder $\Omega_{\rho}^{M_{2}}$, we obtain

$$
\int_{\Omega_{\rho}^{M_{2}}}\left\{\hat{p}_{n} \triangle \overline{\hat{p}_{n}}+\nabla \hat{p}_{n} \nabla \overline{\hat{p}_{n}}\right\} d V=\int_{\Omega_{\rho}^{M_{2}}} k_{2}^{2}\left|\hat{p}_{n}\right|^{2}+\left|\nabla \hat{p}_{n}\right|^{2} d V \in \boldsymbol{R} .
$$

Hence, it follows that

$$
\operatorname{Im}\left\{\int_{C_{\rho}^{M_{2}}} \hat{p}_{n} \frac{\overline{\partial \hat{p}_{n}}}{\partial v} d \sigma\right\}=\operatorname{Im}\left\{-\int_{\Gamma_{\rho}^{d^{+}}} \hat{p}_{n} \frac{\overline{\partial \hat{p}_{n}}}{\partial v} d \sigma\right\} .
$$


Now, applying (36) and (37) to (33) and considering the transmission conditions on $\Gamma_{\rho}^{d}$, we obtain

$$
\begin{aligned}
& \lim _{\rho \rightarrow \infty}\left\{\frac{1}{\rho_{1}} \int_{C_{\rho}^{M_{1}}}\left(\left|\frac{\partial \hat{p}_{n}}{\partial v}\right|^{2}+a_{n}^{2}\left|\hat{p}_{n}\right|^{2}\right) d \sigma+\frac{1}{\rho_{2}} \int_{C_{\rho}^{M_{2}}}\left(\left|\frac{\partial \hat{p}_{n}}{\partial v}\right|^{2}+a_{n}^{2}\left|\hat{p}_{n}\right|^{2}\right) d \sigma\right\} \\
= & \frac{2 a_{n}}{\rho_{1}} \int_{\partial \Omega} \hat{p}_{n} \frac{\partial \hat{p}_{n}}{\partial v} d \sigma .
\end{aligned}
$$

From (38), (32) follows immediately.

For $n>N$, we have $a_{n}=i\left|a_{n}\right|$ and

$$
\hat{p}_{n} \sim H_{0}^{(1)}\left(a_{n} r\right)=O\left(\frac{e^{-\left|a_{n}\right| r}}{r^{1 / 2}}\right) \text { as } r \longrightarrow \infty .
$$

As a result, we get

$$
\sum_{n=N+1}^{\infty} \int_{C_{\rho}^{M_{\alpha}}}\left|\hat{p}_{n}\right|^{2}=O(1) \text { as } \rho \longrightarrow \infty, \alpha=1,2 .
$$

By (8), (32) and (39), the lemma is proved.

\section{Lemma 4.2.}

$$
\begin{aligned}
& \int_{C_{\rho}^{M_{1}}}\left[G(\xi, \zeta ; x, z) \frac{\partial u}{\partial v}(\xi, \zeta)-p(\xi, \zeta) \frac{\partial G(\xi, \zeta ; x, z)}{\partial v}\right] d \sigma \\
+ & \frac{\rho_{1}}{\rho_{2}} \int_{C_{\rho}^{M_{2}}}\left[G(\xi, \zeta ; x, z) \frac{\partial p}{\partial v}(\xi, \zeta)-p(\xi, \zeta) \frac{\partial G(\xi, \zeta ; x, z)}{\partial v}\right] d \sigma \longrightarrow 0 \\
& \text { as } \rho \longrightarrow \infty, \text { for }(x, z) \in\left(\Omega_{\rho}^{M_{1}} \backslash \bar{\Omega}\right) \cup \Omega_{\rho}^{M_{2}} .
\end{aligned}
$$

Proof. Recall (24) that $G\left(r, z, z_{s}\right)=-\frac{i}{4} \sum_{n=0}^{\infty} \psi_{n}(z) \psi_{n}\left(z_{s}\right) H_{0}^{(1)}\left(a_{n} r\right)$.

Define new variables

$$
\begin{aligned}
\hat{G}\left(r, z, z_{s}\right) & :=-\frac{i}{4} \sum_{n=0}^{\infty} a_{n} \psi_{n}(z) \psi_{n}\left(z_{s}\right) H_{0}^{(1)}\left(a_{n} r\right), \\
G_{N}\left(r, z, z_{s}\right) & :=-\frac{i}{4} \sum_{n \leq N} \psi_{n}(z) \psi_{n}\left(z_{s}\right) H_{0}^{(1)}\left(a_{n} r\right), \\
\hat{G}_{N}\left(r, z, z_{s}\right) & :=-\frac{i}{4} \sum_{n \leq N} a_{n} \psi_{n}(z) \psi_{n}\left(z_{s}\right) H_{0}^{(1)}\left(a_{n} r\right), \\
G_{N}^{\prime}\left(r, z, z_{s}\right) & :=-\frac{i}{4} \sum_{n>N} \psi_{n}(z) \psi_{n}\left(z_{s}\right) H_{0}^{(1)}\left(a_{n} r\right), \\
\hat{G}_{N}^{\prime}\left(r, z, z_{s}\right) & :=-\frac{i}{4} \sum_{n>N} a_{n} \psi_{n}(z) \psi_{n}\left(z_{s}\right) H_{0}^{(1)}\left(a_{n} r\right),
\end{aligned}
$$


where $a_{n}>0$ if $n \geq N$ and $i a_{n}<0$ if $n>N$.

Considering the asymptotic behavior of Hankel function

$$
H_{0}^{(1)}(r) \approx\left(\frac{2}{\pi r}\right)^{1 / 2} e^{i(r-\pi / 4)} \text { as } r \longrightarrow \infty,
$$

we have the following estimates:

$$
\begin{aligned}
G_{N}^{\prime} & =O\left(\frac{1}{\sqrt{r}}\right), \\
\hat{G}_{N}^{\prime} & =O\left(\frac{1}{\sqrt{r}}\right), \\
\frac{\partial}{\partial r} G_{N}^{\prime} & =O\left(\frac{1}{r^{3 / 2}}\right), \\
\frac{\partial}{\partial r} \hat{G}_{N}^{\prime} & =O\left(\frac{1}{r^{3 / 2}}\right), \\
\frac{\partial}{\partial r} G_{N}-i \hat{G}_{N} & =-\frac{i}{4} \sum_{n \leq N} \psi_{n}(z) \psi\left(z_{s}\right)\left[\frac{\partial}{\partial r} H_{0}^{(1)}\left(a_{n} r\right)-i a_{n} H_{0}^{(1)}\left(a_{n} r\right)\right] \\
& =O\left(\frac{1}{r^{3 / 2}}\right) \text { as } r \longrightarrow \infty .
\end{aligned}
$$

For $(x, z) \in \Omega_{\rho}^{M_{1}} \backslash \bar{\Omega}$, we rewrite the integrals in lemma 4.2 as

$$
\begin{aligned}
& \int_{C_{\rho}^{M_{1}}}\left[G(\xi, \zeta ; x, z) \frac{\partial p}{\partial v}(\xi, \zeta)-p \frac{\partial G}{\partial v}\right] d \sigma \\
& +\frac{\rho_{1}}{\rho_{2}} \int_{C_{\rho}^{M_{2}}}\left[G(\xi, \zeta ; x, z) \frac{\partial p}{\partial v}(\xi, \zeta)-p \frac{\partial G}{\partial v}\right] d \sigma \\
= & \int_{C_{\rho}^{M_{1}}}\left\{\left[G(\xi, \zeta ; x, z) \frac{\partial p}{\partial v}(\xi, \zeta)-i \hat{G} p\right]-p\left(\frac{\partial G}{\partial v}-i \hat{G}\right)\right\} d \sigma \\
+ & \frac{\rho_{1}}{\rho_{2}} \int_{C_{\rho}^{M_{2}}}\left\{\left[G(\xi, \zeta ; x, z) \frac{\partial p}{\partial v}(\xi, \zeta)-i \hat{G} p\right]-p\left(\frac{\partial G}{\partial v}-i \hat{G}\right)\right\} d \sigma .
\end{aligned}
$$

By lemma 4.1, (45) and the Cauchy-Schwarz inequality, we have

$$
\left|\int_{C_{\rho}^{M_{\alpha}}}\left(\frac{\partial G}{\partial r}-i \hat{G}\right) p d \sigma\right| \rightarrow 0 \text { as } \rho \rightarrow \infty, \quad \alpha=1,2 .
$$

As for the first term in each integral on the right-hand side of (46), we replace 
$p, G$ and $\hat{G}$ with their normal mode expansions and apply lemma 2.4 to obtain

$$
\begin{aligned}
& \left|\int_{C_{\rho}^{M_{1}}}\left(G \frac{\partial p}{\partial r}-i \hat{G} p\right) d \sigma+\frac{\rho_{1}}{\rho_{2}} \int_{C_{\rho}^{M_{2}}}\left(G \frac{\partial p}{\partial r}-i \hat{G} p\right) d \sigma\right| \\
\leq & \frac{1}{4} \sum_{n=0}^{\infty}\left|\int_{0}^{2 \pi}\left\{\psi_{n}\left(z_{s}\right) H_{0}^{(1)}\left(a_{n} \rho\right)\left[\frac{\partial p_{n}}{\partial r}(\rho, \theta)-i a_{n} p_{n}(\rho, \theta)\right] \rho\right\} d \theta\right| \\
& \cdot\left\{\int_{0}^{d}\left|\psi_{n}(\zeta)\right|^{2} d \zeta+\frac{\rho_{1}}{\rho_{2}} \int_{d}^{h}\left|\psi_{n}(\zeta)\right|^{2} d \zeta\right\} \\
= & \frac{1}{4} \sum_{n=0}^{\infty}\left|\int_{0}^{2 \pi}\left\{\psi_{n}\left(z_{s}\right) H_{0}^{(1)}\left(a_{n} \rho\right)\left[\frac{\partial p_{n}}{\partial r}(\rho, \theta)-i a_{n} p_{n}(\rho, \theta)\right] \rho\right\} d \theta\right| .
\end{aligned}
$$

In (48), the horizontal distance $r=\left|x_{s}-\xi\right|$, where $\left(x_{s}, z_{s}\right)$ is the source location and $(\xi, \zeta)$ is a point on $C_{\rho}^{M_{\alpha}}, \alpha=1$ or 2 . Similar notation is used in the rest of the proof. Note that we have applied (25) and (26) to get the last equality.

Applying the radiation condition (9), and considering the asymptotic behavior of $H_{0}^{(1)}\left(a_{n} r\right)$ as stated in (40), we may conclude from (48) that

$$
\lim _{\rho \rightarrow \infty}\left\{\int_{C_{\rho}^{M_{1}}}\left(G \frac{\partial p}{\partial r}-i \hat{G} p\right) d \sigma+\frac{\rho_{1}}{\rho_{2}} \int_{C_{\rho}^{M_{1}}}\left(G \frac{\partial p}{\partial r}-i \hat{G} p\right) d \sigma\right\}=0 .
$$

By a similar argument, we can also show the same result for $(x, z) \in \Omega_{\rho}^{M_{2}}$ From lemma 4.2, we get the following representation theorem:

Theorem 4.1. Let $p \in C^{2}\left(M_{1} \backslash \bar{\Omega}\right) \cap C\left(M_{1} \backslash \Omega\right) \cap C^{2}\left(M_{2}\right)$ be a solution to the problem $(1) \sim(9)$, then

$$
p(x, z)=\left\{\begin{aligned}
& \int_{\partial \Omega}\left[p(\xi, \zeta) \frac{\partial G}{\partial v}(x, z ; \xi, \zeta)-G(x, z ; \xi, \zeta) \frac{\partial p}{\partial v}(\xi, \zeta)\right] d \sigma \\
& i f(x, z) \in M_{1} \backslash \bar{\Omega} \\
& \frac{\rho_{1}}{\rho_{2}} \int_{\partial \Omega}\left[p(\xi, \zeta) \frac{\partial G}{\partial v}(x, z ; \xi, \zeta)-G(x, z ; \xi, \zeta) \frac{\partial p}{\partial v}(\xi, \zeta)\right] d \sigma \\
& \text { if }(x, z) \in M_{2} .
\end{aligned}\right.
$$

\section{A uniqueness theorem}

We want to show that if $p \in C^{2}\left(M_{1} \backslash \bar{\Omega}\right) \cap C\left(R_{h}^{3} \backslash \Omega\right) \cap C^{2}\left(M_{2}\right)$ is a solution to the problem $(1) \sim(9)$ with homogeneous data $g(x, z)=0$ and $k_{1}, k_{2}$ are chosen such 
that $a_{n} \neq 0$ for $n=0,1,2, \ldots$, then $p \equiv 0$ in $R_{h}^{3}$. We model our proof on that of $\mathrm{Xu}$ 's proof in the case of the one-layer problem. See [2] in this regard.

Lemma 5.1. If $p \in C^{2}\left(M_{1} \backslash \bar{\Omega}\right) \cap C\left(R_{h}^{3} \backslash \Omega\right) \cap C^{2}\left(M_{2}\right)$ is a solution to the problem (1) - (9) with homogeneous data $g(x, z)=0$ and $k_{1}, k_{2}$ are chosen such that $a_{n} \neq 0$ for $n=0,1, \ldots$ Then for any $\rho>0$ such that $\Omega \subset \subset \Omega_{\rho}$, we have

$$
\operatorname{Im}\left\{\int_{0}^{2 \pi} \sum_{n=0}^{\infty}<p_{n}, \frac{\partial \overline{p_{n}}}{\partial r}>\left._{*}\right|_{r=\rho} \rho d \theta\right\}=0 .
$$

Proof. By Green's theorem and (1), we have

$$
\begin{aligned}
0 & =\int_{\partial \Omega \cup C_{\rho}^{M_{1}} \cup \Gamma_{\rho}^{d^{-}}} p \frac{\partial \bar{p}}{\partial v}-\bar{p} \frac{\partial p}{\partial v} d \sigma \\
& =\int_{C_{\rho}^{M_{1}}} p \frac{\partial \bar{p}}{\partial r}-\bar{p} \frac{\partial p}{\partial r} d \sigma+\int_{\Gamma_{\rho}^{d^{-}}} p \frac{\partial \bar{p}}{\partial z}-\bar{p} \frac{\partial p}{\partial z} d \sigma \\
0 & =\int_{C_{\rho}^{M_{2}} \cup \Gamma_{\rho}^{d^{+}}} p \frac{\partial \bar{p}}{\partial v}-\bar{p} \frac{\partial p}{\partial v} d \sigma \\
& =\int_{C_{\rho}^{M_{2}}} p \frac{\partial \bar{p}}{\partial r}-\bar{p} \frac{\partial p}{\partial r} d \sigma-\int_{\Gamma_{\rho}^{d^{+}}} p \frac{\partial \bar{p}}{\partial z}-\bar{p} \frac{\partial p}{\partial z} d \sigma .
\end{aligned}
$$

Multiplying and adding, i.e. $\frac{1}{\rho_{1}} \times(53)+\frac{1}{\rho_{2}} \times(54)$, we obtain:

$$
0=\operatorname{Im}\left\{\int_{C_{\rho}^{M_{1}}} \frac{1}{\rho_{1}} p \frac{\partial \bar{p}}{\partial r} d \sigma\right\}+\operatorname{Im}\left\{\int_{C_{\rho}^{M_{2}}} \frac{1}{\rho_{2}} p \frac{\partial \bar{p}}{\partial r} d \sigma\right\} .
$$

For $\rho$ big enough, we may replace $p$ in (55) with its normal mode representation (8) to get

$$
0=\operatorname{Im}\left\{\int_{0}^{2 \pi} \sum_{n=0}^{\infty} \sum_{m=0}^{\infty} \frac{1}{\rho_{1}}<\psi_{n}, \psi_{m}>_{*} p_{n}(\rho, \theta) \frac{\partial \bar{p}_{m}}{\partial r}(\rho, \theta) \rho d \theta\right\} .
$$

By applying the orthonormality of $\left\{\psi_{n}\right\}$ given in lemma 2.3 to (56), the lemma is proved.

Lemma 5.2. Under the assumptions of lemma 5.1, we have

$$
p=O\left(\frac{e^{-\left|a_{N+1}\right| r}}{r^{1 / 2}}\right) \text { as } r \longrightarrow \infty .
$$

Proof. Refer to the proof of lemma 2.2 in [2]. 
Lemma 5.3. Let $n_{x}=\left(n_{1}, n_{2}, n_{3}\right)$ be the outward unit normal vector of $\partial \Omega$ at $(x, z)$, $n_{x}{ }^{\prime}:=\left(n_{1}, n_{2}\right)$, and $x:=(x, y)$.

If $x \cdot v_{x}^{\prime} \geq 0$ for all $(x, z) \in \partial \Omega$, and $p$ satisfies the assumption in lemma 5.1, then for $(x, z)$ in $\left(M_{1} \backslash \bar{\Omega}\right) \cup M_{2}, p(x, z)$ satisfies

$$
\frac{\partial p}{\partial x}=0, \quad \frac{\partial p}{\partial y}=0
$$

Proof. Let $\nabla^{\prime}:=\left(\frac{\partial}{\partial x}, \frac{\partial}{\partial y}\right), v=\left(v_{1}, v_{2}, v_{3}\right)$ denote the outward unit normal vector of an oriented surface, and $v^{\prime}:=\left(v_{1}, v_{2}\right)$. By a calculation similar to that of lemma 2.3 in [2], we have

$$
\begin{aligned}
A(\rho):= & \int_{\partial\left(\Omega_{\rho}^{M_{1}} \backslash \Omega\right)} x \cdot\left[\frac{\partial \bar{p}}{\partial v} \nabla^{\prime} p+\frac{\partial p}{\partial v} \nabla^{\prime} \bar{p}-|\nabla p|^{2} v^{\prime}\right] d \sigma \\
= & 2 \int_{\Omega_{\rho}^{M_{1}} \backslash \Omega}\left|\nabla^{\prime} p\right|^{2} d V-k_{1}^{2} \int_{C_{\rho}^{M_{1}}} \rho|p|^{2} d \sigma \\
& -2\left[\int_{C_{\rho}^{M_{1}}} \bar{p} \frac{\partial p}{\partial v} d \sigma+\int_{\Gamma_{\rho}^{d^{-}}} \bar{p} \frac{\partial p}{\partial v} d \sigma\right] .
\end{aligned}
$$

Here we have used the fact that $v^{\prime} \cdot x=\rho$ on $C_{\rho}^{M_{1}}, v^{\prime} \cdot x=0$ on $\Gamma_{\rho}^{0} \cup \Gamma_{\rho}^{d^{-}}$and the pressure release boundary condition (3) of $p$ on $\Gamma_{\rho}^{0}$.

Using the same reasoning, we get

$$
\begin{aligned}
B(\rho):= & \int_{\partial \Omega_{\rho}^{M_{2}}} x \cdot\left[\frac{\partial \bar{p}}{\partial v} \nabla^{\prime} p+\frac{\partial p}{\partial v} \nabla^{\prime} \bar{p}-|\nabla p|^{2} v^{\prime}\right] d \sigma \\
= & 2 \int_{\Omega_{\rho}^{M_{2}}}\left|\nabla^{\prime} p\right|^{2} d V-k_{2}^{2} \int_{C_{\rho}^{M_{2}}} \rho|p|^{2} d \sigma \\
& \left.-2\left[\int_{C_{\rho}^{M_{2}}} \bar{p} \frac{\partial p}{\partial v} d \sigma+\int_{\Gamma_{\rho}^{d^{+}}} \bar{p} \frac{\partial p}{\partial v} d \sigma\right]\right] .
\end{aligned}
$$

Applying the transmission conditions (4) and (5) to $\frac{1}{\rho_{1}} A(\rho)+\frac{1}{\rho_{2}} B(\rho)$ gives

$$
\begin{aligned}
& \frac{1}{\rho_{1}} A(\rho)+\frac{1}{\rho_{2}} B(\rho) \\
= & \frac{2}{\rho_{1}} \int_{\Omega_{\rho}^{M_{1}} \backslash \Omega}\left|\nabla^{\prime} p\right|^{2} d V-\frac{k_{1}^{2}}{\rho_{1}} \int_{C_{\rho}^{M_{1}}} r|p|^{2} d \sigma \\
& -\frac{2}{\rho_{1}} \int_{C_{\rho}^{M_{1}}} \bar{p} \frac{\partial p}{\partial v} d \sigma-\frac{2}{\rho_{2}} \int_{C_{\rho}^{M_{2}}} \bar{p} \frac{\partial p}{\partial v} d \sigma \\
& +\frac{2}{\rho_{2}} \int_{\Omega_{\rho}^{M_{2}} \backslash \Omega}\left|\nabla^{\prime} p\right|^{2} d V-\frac{k_{2}^{2}}{\rho_{2}} \int_{C_{\rho}^{M_{2}}} r|p|^{2} d \sigma .
\end{aligned}
$$


On the other hand, since $p=0$ on $\partial \Omega$, we have $\nabla p=v \frac{\partial p}{\partial v}, \nabla^{\prime} p=v^{\prime} \frac{\partial p}{\partial v}$ on $\partial \Omega$. Also note that $p=0, \nabla^{\prime} p=0$ on $\Gamma_{\rho}^{0}$ and $x \cdot v^{\prime}=0$ on $\Gamma_{\rho}^{d^{-}}$. Using all these facts, we may rewrite $A(\rho)$ as

$$
\begin{aligned}
A(\rho)= & \int_{\partial \Omega} x \cdot v^{\prime}\left|\frac{\partial p}{\partial v}\right|^{2} d \sigma+\int_{C_{\rho}^{M_{1}}} \rho\left[2\left|\frac{\partial p}{\partial r}\right|^{2}-|\nabla p|^{2}\right] d \sigma \\
& +\int_{\Gamma_{\rho}^{d^{-}}} x \cdot\left[\frac{\partial \bar{p}}{\partial v} \nabla^{\prime} p+\frac{\partial p}{\partial v} \nabla^{\prime} \bar{p}\right] d \sigma .
\end{aligned}
$$

Similarly, we also have

$$
B(\rho)=\int_{C_{\rho}^{M_{2}}} \rho\left[2\left|\frac{\partial p}{\partial r}\right|^{2}-|\nabla p|^{2}\right] d \sigma+\int_{\Gamma_{\rho}^{d^{+}}} x \cdot\left[\frac{\partial \bar{p}}{\partial v} \nabla^{\prime} p+\frac{\partial p}{\partial v} \nabla^{\prime} \bar{p}\right] d \sigma .
$$

Noting that $\lim _{z \rightarrow d^{-}} p(x, z)=\lim _{z \rightarrow d^{+}} p(x, z), \forall x \in \boldsymbol{R}^{2}$, we realize

$$
\nabla^{\prime} p\left(x, d^{-}\right)=\nabla^{\prime} p\left(x, d^{+}\right), \forall x \in \boldsymbol{R}^{2} .
$$

By considering (59) and the transmission condition (4) on $\Gamma_{\rho}^{d}$, we get another identity

$$
\begin{aligned}
& \frac{1}{\rho_{1}} A(\rho)+\frac{1}{\rho_{2}} B(\rho) \\
= & \frac{1}{\rho_{1}} \int_{\partial \Omega} x \cdot v^{\prime}\left|\frac{\partial p}{\partial v}\right|^{2} d \sigma+\frac{1}{\rho_{1}} \int_{C_{\rho}^{M_{1}}} \rho\left[2\left|\frac{\partial \bar{p}}{\partial v}\right|^{2}-|\nabla p|^{2}\right] d \sigma \\
& +\frac{1}{\rho_{2}} \int_{C_{\rho}^{M_{2}}} \rho\left[2\left|\frac{\partial p}{\partial r}\right|^{2}-|\nabla p|^{2}\right] d \sigma .
\end{aligned}
$$

Equating the right-hand side of (58) with that of (60), letting $\rho \longrightarrow \infty$ and applying lemma 5.2 yield

$$
\frac{2}{\rho_{1}} \int_{\Omega_{\rho}^{M_{1}} \backslash \Omega}\left|\nabla^{\prime} p\right|^{2} d V+\frac{2}{\rho_{2}} \int_{\Omega_{\rho}^{M_{2}}}\left|\nabla^{\prime} p\right|^{2} d V=\frac{1}{\rho_{1}} \int_{\partial \Omega} x \cdot v^{\prime}\left|\frac{\partial p}{\partial v}\right|^{2} d \sigma .
$$

Noting that $v^{\prime}=-v_{x}^{\prime}$, we can conclude from $x \cdot v_{x}^{\prime} \geq 0$ and (61) that

$$
\frac{2}{\rho_{1}} \int_{\Omega_{\rho}^{M_{1}} \backslash \Omega}\left|\nabla^{\prime} p\right|^{2} d V+\frac{2}{\rho_{2}} \int_{\Omega_{\rho}^{M_{2}}}\left|\nabla^{\prime} p\right|^{2} d V \leq 0
$$

Therefore, we must have $\nabla^{\prime} p=0$. 
Theorem 5.1. Let $\Omega$ be a bounded domain in $M_{1}$ with boundary of class $C^{2}$ such that $\Omega \cap \Gamma=\emptyset$. Let $n_{x}=\left(n_{1}, n_{2}, n_{3}\right)$ be the outward unit normal vector to the boundary $\partial \Omega$ at $(x, z)$ and assume $x \cdot\left(n_{1}, n_{2}\right) \geq 0, \forall(x, z) \in \partial \Omega$.

If $p \in C^{2}\left(M_{1} \backslash \bar{\Omega}\right) \cap C\left(R_{h}^{3} \backslash \Omega\right) \cap C^{2}\left(M_{2}\right)$ is a solution to the problem (1) - (9) with homogeneous boundary data $g=0$ and with $k_{1}, k_{2}$ chosen such that none of the eigenvalues of the problem (1) - (9) is zero, then $p \equiv 0$ in $M_{1} \cup \Gamma \cup M_{2}$.

Proof. By lemma 5.3, we have $\frac{\partial p}{\partial x}=0, \frac{\partial p}{\partial y}=0$ in $M_{1} \backslash \bar{\Omega}$. So $g=0$ on $\partial \Omega$ implies the existence of a connected open set $S \subset M_{1}$ where $p$ vanishes identically. Moreover, $p$ is analytic in $M_{1}$ by the representation formula (50). Therefore, $p \equiv 0$ in $M_{1}$.

Since $p$ vanishes in $M_{1}$, we can conclude that $p \equiv 0$ in $M_{2}$ by considering the representation formula (51).

By the continuity of $p$ in $R_{h}^{3}$, we have $p \equiv 0$ on $\Gamma$ and the theorem follows immediately.

\section{References}

[1] M. Ou. Direct and Inverse Acoustic Scattering Problems for a Class of Threedimensional Waveguides. PhD thesis, University of Delaware, 2001.

[2] Y. Xu. Direct and Inverse Scattering in Shallow Oceans. PhD thesis, University of Delaware, 1991.

[3] Y. Xu. A note on the uniqueness of the propagating solution for acoustic waves in a finite depth ocean. Applicable Analysis, 67(91), 1997.

[4] Y. Xu. Inverse acoustic scattering problems in ocean environment. Computational Acoustics, 7(2), 1999. 